\title{
The research of dynamic stability control system for passenger cars using CarSim and Matlab-Simulink
}

\author{
Ying Yang ${ }^{\mathrm{a}}$, Bulus Titus Dogara and Meng He \\ School of Mechanical Engineering and Automation, Northeastern University, Shenyang, 110189, China
}

\begin{abstract}
Electronic stability control (ESC) systems are well understood with regard to assisting drivers to maintain vehicle control during extreme handling maneuvers or when extreme road conditions are encountered. This paper discusses the development of a vehicle dynamics model and model validation of a certain vehicle in Matlab-Simulink and Carsim. The vehicle dynamics model created in Carsim was validated by comparing simulation results with quasi-static and dynamic test data. The Sine with Dwell maneuver was used to validate the complete vehicle model. The ESC system in this paper was designed to operate in two modes: Yaw Stability Control (YSC) and Roll Stability Control (RSC). The performance of the system was evaluated using dynamic maneuvers like the Sine with Dwell and Fishhook maneuvers. This test was used to evaluate the performance of the Yaw Stability Control mode of the ESC system. This paper describes a model-based design approach for the development of a functional ESC system model. The software used is Carsim and Matlab Simulink.
\end{abstract}

Keywords: ESC; stability control; rollover prevention; vehicle dynamics.

\section{Introduction}

With the substantial presence of vehicles and an increasing emphasis on vehicle safety, there is great need for cost effective methods of rollover mitigation. This is seen in the commercial market with all sizes of vehicles where manufacturers are being held to increasingly higher standards of safety. These systems must be able to accurately detect impending rollover soon enough to activate mitigation hardware without misinterpreting safe vehicle maneuvers as rollover situations.

In extreme maneuvers when the vehicle is operating at the limits of road traction, the vehicle response to driver inputs is different from normal and hence the driver cannot control the vehicle [1-3]. The objective of this study is not to build a comprehensive ESC model that would account for all loss-of-control scenarios. Rather, the ESC model developed here is expected to demonstrate that given a vehicle model, it is possible to construct a simple, functional ESC model which will give comparable performance to actual ESC systems on board the vehicle for certain maneuvers. This allows us to allocate resources to the main task of evaluating the effect of the new component or system on the performance of the ESC-equipped vehicle.

${ }^{\text {a }}$ Corresponding author : yangyang@me.neu.edu.cn

(C) 2016. The authors - Published by Atlantis Press 


\section{Vehicle ESC modeling}

The benefits of using of using ESC are realized only if the model is representative of the actual system [4]. This part discusses the development of a vehicle dynamics model in CarSim. The vehicle model will then be validated by comparing simulation results with experimental test data. Bounce and Roll tests in CarSim will be used to validate the suspension and steering kinematics and compliances. Field test data of the Sine with Dwell maneuver(s) will be used for the vehicle model validation. The vehicle modeled is a 4-door sedan vehicle with a 5.4L V8 engine, automatic transmission. The parameters of the vehicle model created in CarSim are based on various measurements and reference from a certain vehicle technical data page. These parameters were then used to build the CarSim model.

\subsection{ESC system topography}

The ESC system model was built in Simulink and was tuned through co- simulation with CarSim. Parameters such as vehicle speed, steering wheel angle, lateral acceleration, yaw rate and longitudinal slip ratios are obtained from the CarSim vehicle model and are used as inputs to the ESC model.

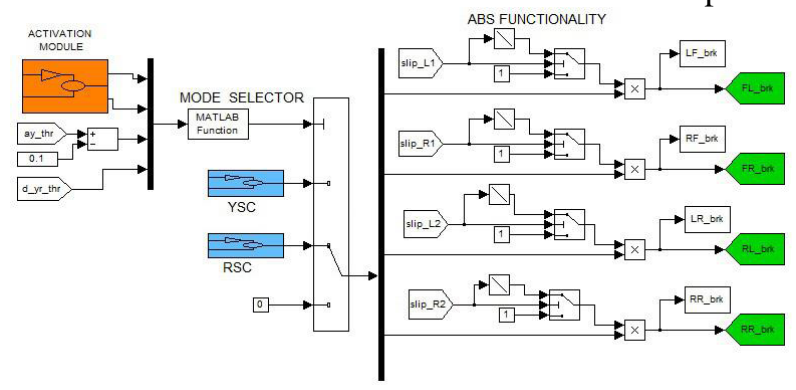

Figure 1. ESC system topography

Fig. 1 shows the main building blocks of the ESC system. The activation module calculates the values of the control variables (lateral acceleration for roll stability and vehicle slip rate for yaw stability) at each time step. The vehicle slip rate is the difference between actual and ideal yaw rates. The ideal yaw rate is the ratio of vehicle lateral acceleration to the vehicle longitudinal velocity. The actual yaw rate is obtained from CarSim.

The values obtained from the activation module are compared to the threshold values of the control variables. The values of in the test data - corresponding to the activation of the ESC system on-board the test vehicle - were used to set the threshold for the ESC model in Simulink.

\subsection{Roll and yaw stability control}

The Roll Stability Control module (shown in Figure 2) contains the control logic for selecting the wheel to be braked and for calculating the magnitude of braking force, so that the vehicle does not rollover. The time delay block is used to simulate the time delay in the brake system - from the time ESC is activated to the time brakes are actually applied. The RSC system brakes the outer front wheel when the lateral acceleration exceeds the threshold value. The steering wheel angle obtained from CarSim is used to determine the direction of turn of the vehicle and accordingly, the outer front wheel is braked. The brake pressures calculated in this module are then passed to CarSim vehicle model. 


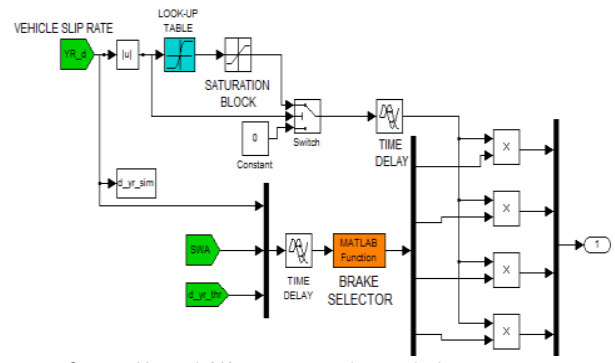

Figure 2. Roll stability control module

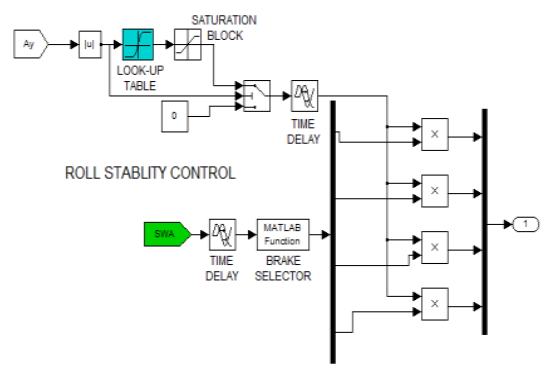

Figure. 3 Yaw stability control modules

Yaw Stability Control module (shown in Figure3) uses differential braking to ensure that the vehicle retains directional stability. The brake pressures calculated in this module then pass through the ABS block (which checks for wheel slip and reduces brake pressure if required) and are then used as inputs to the CarSim vehicle model.

\section{Simulation results}

This chapter contains the results of co-simulation between the vehicle model in CarSim and the ESC model in Matlab-Simulink. The test conditions, model parameters and thresholds used for the tests are also described. The simulation results are then used to evaluate the performance of the ESC system model.

\subsection{Yaw stability performance evaluations}

The sine with dwell (SWD) test is used to evaluate the performance of yaw stability control mode of the ESC system. Figure 4 shows the comparison plots of simulation results and test data - for the BMW 5Series - in the baseline mode (ESC turned OFF) and with ESC. The performance of the ESC system model is comparable to the actual ESC system on board the vehicle, for this particular maneuver. The vehicle slip rate threshold used for the ESC model was 0.18.
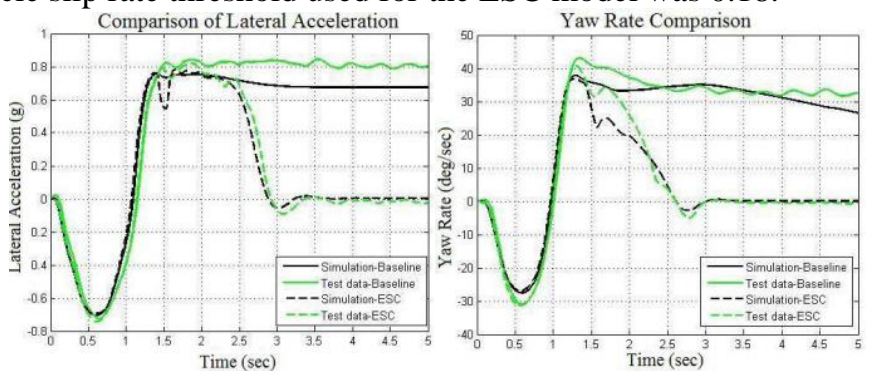

Figure 4. Effects of ESC Intervention

Fig. 4 showed the improvement in lateral stability due to ESC by comparing the response of baseline and ESC equipped vehicles.
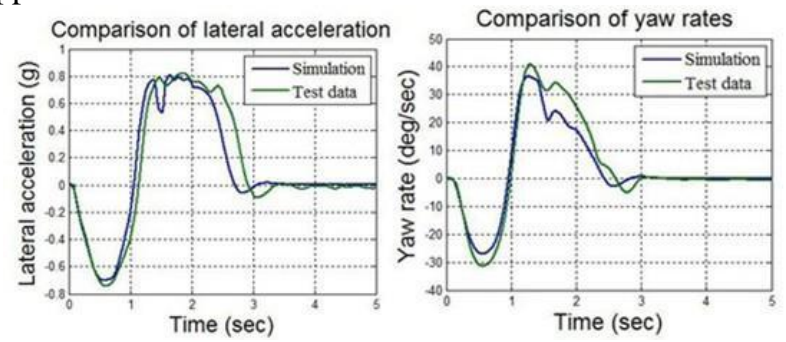

Figure 5. Sine with dwell 
Fig. 5 shows that the test and simulated vehicles have the same steering input and initial speed (90 $\mathrm{km} / \mathrm{h}$ ). Further the lateral acceleration and yaw rate response plots show that the performance of the ESC system model and actual on-board ESC system is also comparable for this maneuver.

The main objective that has to be achieved is that the ESC system model gives performance (in terms of lateral acceleration and yaw rate response) comparable to the system on board the vehicle.
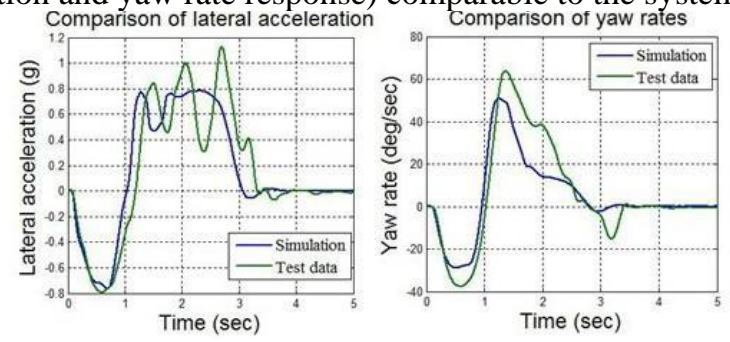

Figure 6. Sine with dwell: 280 degrees.

Fig. 6 shows that even for a severe maneuver, the ESC system model gives satisfactory performance. The plots of lateral acceleration and yaw rate show that the ESC model performance is comparable to the actual on-board system. The discussion until now was limited to the Yaw Stability Mode of the ESC system and the sine with dwell maneuver was used to evaluate ESC system performance. The following discussion is for the Roll Stability mode of the ESC system.

\subsection{ESC system performance evaluations}

For the YSC mode, test data was available and hence comparisons were made with the ESC system onboard the vehicle. However, there was no data for the ESC system operating in the RSC mode. Further, the vehicle model represents the car with only the driver in it and hence all of the geometric and inertia data for the vehicle is for this loading condition. Accordingly, a new dataset containing the car with a high CG was created in CarSim and the ESC roll stability controller was tuned to stabilize this particular vehicle model. The lateral acceleration threshold for the RSC controller was set to 0.7 while the vehicle slip rate threshold was same as that used in YSC (0.18).

The certain car with high CG was found to rollover for an initial speed of $82 \mathrm{~km} / \mathrm{h}$ at the entrance to the Fishhook maneuver. This initial speed was used to compare the response of the baseline vehicle and the vehicle with the ESC system model.

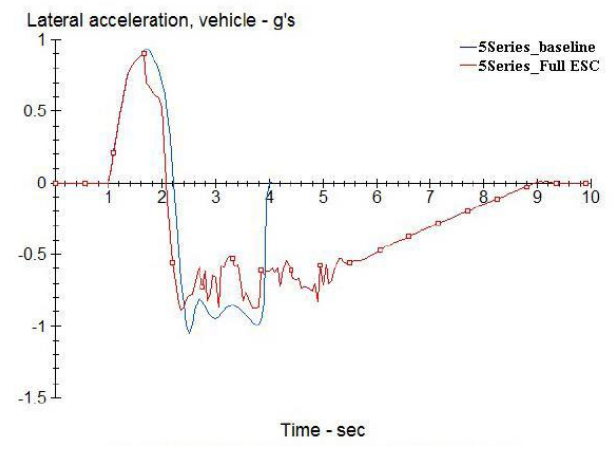

Figure 7. Fishhook $(82 \mathrm{~km} / \mathrm{h})$ : lateral acceleration

Fig. 7 shows the comparison plots of various parameters for the vehicle with and without ESC. It is observed that the ESC system prevents rollover by braking the appropriate front wheels. The baseline vehicle rolls over approximately four seconds after the start of the maneuver and hence the data-points for the baseline vehicle are only until four seconds. The roll rate and roll angle increase rapidly as the vehicle goes into rollover. 


\section{Conclusion}

The simulation results for the yaw stability mode showed that the ESC system model gives comparable performance to the actual system on board the vehicle for those particular test conditions. A fictitious vehicle model was created for evaluating the roll stability mode of the ESC system model. The ESC system was seen to prevent the vehicle model from rolling over. Thus, the above results show that the simple, functional ESC system model improves both lateral and roll stability of the vehicle. Comparison between the vehicle with and without ESC showed that the ESC system improved the roll stability of the vehicle. The parameters which could be used to tune the model for a different vehicle were listed and the tuning procedure was explained in brief.

\section{References}

1. Manning, W.J. and D.A. Crolla, A review of yaw rate and sideslip controllers for passenger vehicles. Transactions of the Institute of Measurement and Control, 2007. 29(2): p. 117-135.

2. Esmailzadeh, E., A. Goodarzi, and G.R. Vossoughi, Optimal yaw moment control law for improved vehicle handling. Mechatronics, 2003. 13(7): p. 659-675.

3. Zheng, S., H. Tang, Z. Han, and Y. Zhang, Controller design for vehicle stability enhancement. Control Engineering Practice, 2006. 14(12): p. 1413-1421.

4. Eslamian, M., G. Alizadeh, and M. Mirzaei, Optimization-based non-linear yaw moment control law for stabilizing vehicle lateral dynamics. Proceedings of the Institution of Mechanical Engineers, Part D: Journal of Automobile Engineering, 2007. 221-233.

5. Abe, M., Y. Kano, K. Suzuki, Y. Shibahata, and Y. Furukawa, Side-slip control to stabilize vehicle lateral motion by direct yaw moment. JSAE Review, 2001. 22(4): p. 413-419.

6. 47. Ungoren, A.Y., H. Peng, and H.E. Tseng, A study on lateral speed estimation methods. Int. J. of Vehicle Autonomous Systems 2004. 2(1/2): p. 126 - 144.

7. Imsland, L., H.F. Grip, T.A. Johansen, T.I. Fossen, J.C. Kalkkuhl, and A. Suissa, Nonlinear Observer for Vehicle Velocity with Friction and Road Bank Angle Adaptation-Validation and Comparison with an Extended Kalman Filter. SAE Paper 2007-01-0808 2007. 